\title{
Palmprint Verification Using Circular Gabor Filter
}

\author{
Azadeh Ghandehari ${ }^{1}$ and Reza Safabakhsh ${ }^{2}$ \\ ${ }^{1}$ Faculty of Engineering \& Technology, Islamic Azad University (Saveh Branch) \\ ${ }^{2}$ Computer Engineering Department, Amirkabir University of Technology \\ (Tehran Polytechnic) \\ ghandehari@iauscd.ir, safa@aut.ac.ir
}

\begin{abstract}
Recently, researchers have been paying an excessive amount of attention to biometric palmprint which has gained popularity and received significant prominence due to its high stability and uniqueness. In this study, two certain filters have been taken into consideration, namely Gabor filter and Circular Gabor filter which are used to obtain feature information from two distinguishing regions of interest, square and inscribed circle areas as the central part sub-images and the two palmprint images are compared with one another in terms of their hamming distance. The outcomes of the experiment gave an indication to the fact that circular Gabor Filter had a comparatively better performance than traditional one in extracting distinct feature information.
\end{abstract}

Keywords: Biometrics, Feature extraction, Gabor filter, Texture analysis.

\section{Introduction}

A plentitude of distinguishing traits that have a wide variety of usage for personal authentication exist, which include fingerprint, face, voice, iris, hand geometry and so forth. Palmprint can be undoubtedly referred to as one of the highly reliable means of making a distinction between two individuals, since the print patterns are not duplicated even in monozygotic twins [1]. More significantly, the details of these patterns are perpetual and the rich structures of the palmprint offer plenty of helpful information for the purpose of recognizing one individual from others [2-4].

Recently, most researches in biometrics have been focused on palmprint authentication, and many approaches have been developed. According to the operating space in which palmprint features extracted, those approaches can be categorized into spatial domain and frequency domain. Due to the type of palmprint features, the above mentioned domains can be categorized into structural feature-based approaches and statistical feature-based approaches. The first approach is a key strategy to extracting structural information, like principal lines and creases, from the palm for recognition [7-11]. The second approach indicates the works that appear in the literature, including eigenpalm [5], fisherpalms [11], global texture energy [13], Gabor filters [13], Fourier Transform [6], and local texture energy [15]. Each approach demonstrates its strengths and weaknesses, and the choice depends on the temperament of application: operational mode, processing speed, memory storage and quality of the image acquired. Generally, a palmprint has some principal lines, wrinkles, singular points and 
minutiae. Some algorithms such as the stack filter [17] can extract the principal lines. However, these principal lines do not contribute adequately to high accuracy because of their similarity amongst different people. Thus, wrinkles play an important role in palmprint authentication. However, in low-resolution palmprint images, some images do not have clear wrinkles. So, in this paper texture analysis has been applied to palmprint authentication.

\section{Overview of System Architecture}

The proposed system is divided into two phases, namely the enrollment and verification, as shown in Fig 1.

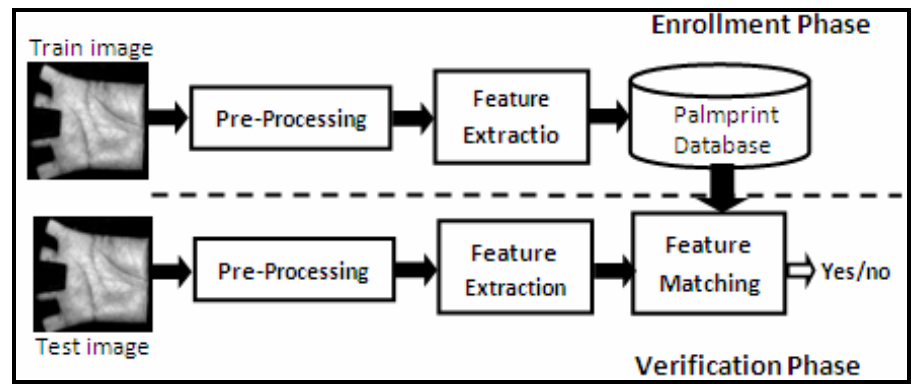

Fig. 1. Block diagram of the proposed palmprint verification phases

The important tasks in the system include the pre-processing, feature extraction and feature matching. In the pre-processing stage, the alignment and orientation of the hand images are corrected for use in the successive tasks. In the feature extraction stage, the most discriminating features of the palms are extracted for representation, and finally in the feature matching stage, comparisons are performed and decisions are made to find out whether two palmprint features are from the same person. The details of each of these components are discussed in the subsequent sections.

\subsection{Pre-processing}

In pre-processing stage, it is necessary to obtain a sub-image from palmprint image and to eliminate the variations caused by rotation and translation. There are two common approaches for the processing of palm images, which differ from each other because of the shape of the sub-image. In the first one, called square-based approach, the sub-images are obtained by cutting a square from the main image, while in the second one the sub-images are extracted by a circular cutting of the main palm image; his is called inscribed circle-based approach. There are many articles reporting the use of square-based approach whereas the inscribed circle-based approach has hardly attracted the attention of researchers. In this paper, we employed both approaches to create two different data bases for feature extractions. 


\subsubsection{Square-Based Approach}

The five main steps of palmprint image preprocessing to extract a square with the fixed size from predefined position under the coordinate system are as follows (see Fig. 2):

Step 1: Apply a low-pass filter, such as Gaussian, to the original image. Then use a threshold to convert this original image into a binary image as shown in Fig. 2(b).

Step 2: Extract the boundaries of the holes between fingers using a boundary-tracking algorithm. The start points, $\left(S x_{i}, S y_{i}\right)$ and end points, $\left(E x_{i}, E y_{i}\right)$ of the holes are then marked in the process (see Fig. 2(c)).

Step 3: Calculate the center of gravity $\left(C x_{i}, C y_{i}\right)$ of the holes. Then construct a line that passes through $\left(C x_{i}, C y_{i}\right)$ and the midpoint of $\left(S x_{i}, S y_{i}\right)$ and $\left(E x_{i}, E y_{i}\right)$. Based on these lines, two key points $\left(K_{1}, K_{2}\right)$ can easily be detected (see Fig. 2(d)).

Step 4: Line up $K_{1}$ and $K_{2}$ to get the Y-axis of the palmprint coordinate system and make a line through their midpoint which is perpendicular to the $\mathrm{Y}$-axis, to determine the origin of the coordinate system (see Fig. 2(e)). This coordinate system can align different palmprint images.

Step 5: Extract a sub-image with the fixed size on the basis of coordinate system, which is located at the certain part of the palmprint for feature extraction (see Fig. 2(f)).

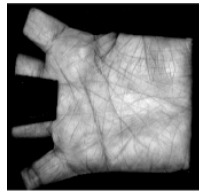

(a)

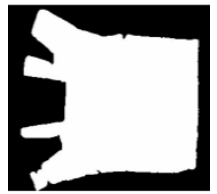

(b)

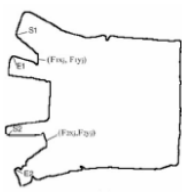

(c)

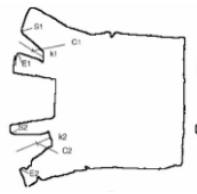

(d)

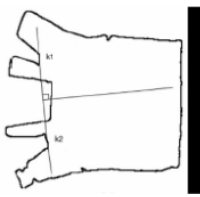

(e)

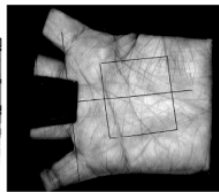

(f)

Fig. 2. Main steps of preprocessing: (a)original image, (b)binary image, (c)boundary tracking, (d)key points (k1 and k3) detecting, (e)the coordinate system and (f)the central part of a palm

\subsubsection{Inscribed Circle-Based Approach}

In contrast to the square-based approach, this approach is based on an inscribed circle to extract the central part of a palmprint; therefore a round image is obtained rather than a square one. The followings describe the details of this approach (Fig. 3):

Step 1: convert the gray scale image into a binary image. Then, smooth the boundary between the white and black pixel and obtain the contour of a palmprint (see Fig. 3(c)).

Step 2: calculate the biggest inscribed circle for the contour of the palmprint and obtain its center and radius. Because different palms are different sized, the radii may be different (see Fig. 3(c)).

Step 3: once the circle is determined, the extraction only invokes obtaining all pixels inside the circle (see Fig. 3(d)). 


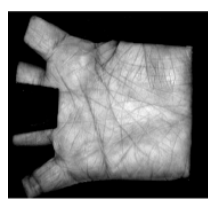

(a)

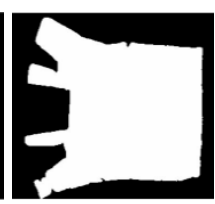

(b)

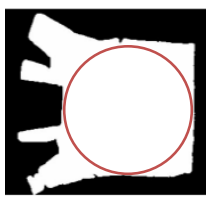

(c)

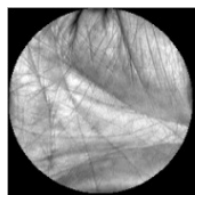

(d)

Fig. 3. Main steps of preprocessing: (a)original image, (b)binary image, (c)biggest inscribed circle and (d) circle area central part sub-image

\subsubsection{Comparison between Two Approaches}

There are cases in which a palmprint cannot be segmented properly using the squarebased approach while it can be done so using the circle-based approach. Fig. 4 shows a sample that the ring finger cannot be placed correctly due to its extreme darkness which cannot be separated from the background. As a result, this palmprint cannot be correctly segmented using the square-based approach. On the other hand, by using the circle-based segmentation approach, the central part palmprint sub-image can be extracted successfully (Fig. 4(c).)

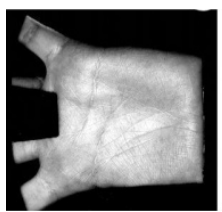

(a)

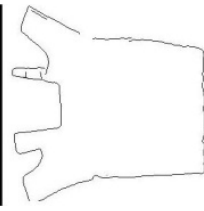

(b)

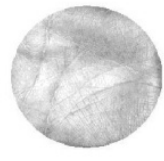

(c)

Fig. 4. Palmprint central part sub-image extraction for special palmprint

The circle-based approach can extract a larger area for the feature extraction, making it possible to include more features. It is worth noting that though the circle-based approach provides a larger surface area for the palmprint feature extraction, it takes longer for feature extraction. Hence, the conclusion is that square-based approach has the advantage of faster processing on the feature extraction while circle-based approach has more features obtained.

Samples from the same palm may have different circle sizes, but the difference is usually within 2-3 pixels, which does not cause a problem for palmprint matching. Different palms may have different circle sizes. It provides a useful means on palmprint classification, i.e. use the circle size as the first step of the classification criteria.

\subsection{Palmprint Feature Extractions}

In this section, the extraction of texture features has been fulfilled through using two types of Gabor filters. The first type of Gabor filter, the conventional one, is used in the texture analysis and the second type, the improved version of the conventional one, is rotation invariant which has been suggested for feature extraction. 


\subsubsection{Traditional 2D Gabor Filter}

The Gabor filter is an effective tool for texture analysis, and has the following general form:

$$
G(x, y, \theta, u, \sigma)=\frac{1}{2 \pi \sigma^{2}} \exp \left\{-\frac{x^{2}+y^{2}}{2 \sigma^{2}}\right\} \times \exp \{2 \pi i u(x \operatorname{Cos} \theta+y \operatorname{Sin} \theta)\}
$$

where $i=\sqrt{-1} ; u$ is the frequency of the sinusoidal wave; $\theta$ controls the orientation of the function and $\sigma$ is the standard deviation of the Gaussian envelope.

Gabor function, $G(x, y, \theta, u, \sigma)$ with a special set of parameters $(\sigma, \theta, u)$, is transformed into a discrete Gabor filter $G[x, y, \theta, u, \sigma]$. The parameters are chosen from 12 sets of parameters listed in Table 1. In this paper, the parameter selection is similar to [12]. In order to provide more robustness to brightness, the Gabor filter is turned to zero DC (direct current) with the application of the following formula:

$$
\tilde{G}[x, y, \theta, u, \sigma]=G[x, y, \theta, u, \sigma]-\frac{\sum_{i=-n}^{n} \sum_{j=-n}^{n} G[i, j, \theta, u, \sigma]}{(2 n+1)^{2}}
$$

where $(2 n+1)^{2}$ is the size of the filter. In fact, the imaginary part of the Gabor filter automatically has zero DC because of odd symmetry. This adjusted Gabor filter will convolute with a sub-image defined in Section 2.1. The sample point in the filtered image is coded to two bits, $\left(b_{r}, b_{i}\right)$ by the following inequalities,

$$
\begin{array}{lll}
b_{r}=1 \text { if } & \operatorname{Re}[\tilde{G}[x, y, \theta, u, \sigma] * I] \geq 0 \\
b_{r}=0 \text { if } & \operatorname{Re}[\tilde{G}[x, y, \theta, u, \sigma] * I]<0 \\
b_{i}=1 \text { if } & \operatorname{Im}[\tilde{G}[x, y, \theta, u, \sigma] * I] \geq 0 \\
b_{i}=0 \text { if } & \operatorname{Im}[\tilde{G}[x, y, \theta, u, \sigma] * I]<0
\end{array}
$$

where I is the sub-image of a palmprint. Using this coding method, only the phase information in palmprint images is stored in the feature vector. Fig. 5 shows the features generated by the 12 filters listed in Table 1 .

Table 1. The traditional Gabor filter's parameters and their definitions

\begin{tabular}{cllcll}
\hline Levels & No & Sizes & $\theta$ & F & $\sigma$ \\
\hline 1 & 1 & $9 \times 9$ & 0 & 0.3666 & 1.4045 \\
& 2 & $9 \times 9$ & 45 & 0.3666 & 1.4045 \\
& 3 & $9 \times 9$ & 90 & 0.3666 & 1.4045 \\
& 4 & $9 \times 9$ & 135 & 0.3666 & 1.4045 \\
\hline 2 & 5 & $17 \times 17$ & 0 & 0.1833 & 2.8090 \\
& 6 & $17 \times 17$ & 45 & 0.1833 & 2.8090 \\
& 7 & $17 \times 17$ & 90 & 0.1833 & 2.8090 \\
& 8 & $17 \times 17$ & 135 & 0.1833 & 2.8090 \\
\hline 3 & 9 & $35 \times 35$ & 0 & 0.1833 & 2.8090 \\
& 10 & $35 \times 35$ & 45 & 0.1833 & 2.8090 \\
& 11 & $35 \times 35$ & 90 & 0.1833 & 2.8090 \\
& 12 & $35 \times 35$ & 135 & 0.1833 & 2.8090 \\
\hline
\end{tabular}




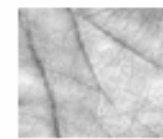

(a)

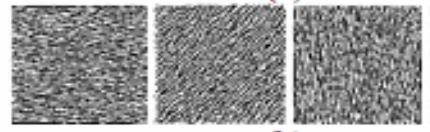

(b)

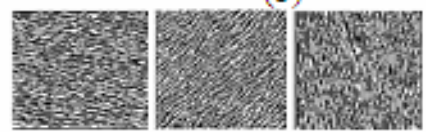

(c)

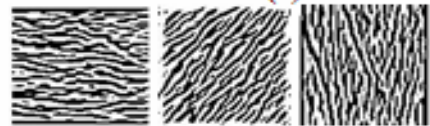

(d)
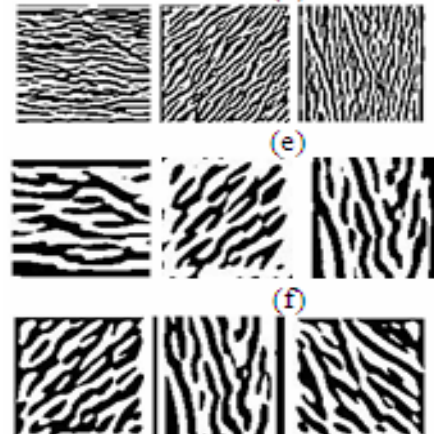

e)
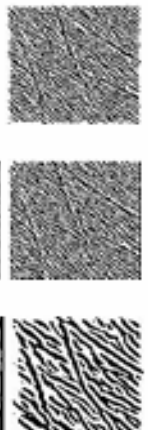

s.'.

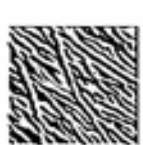

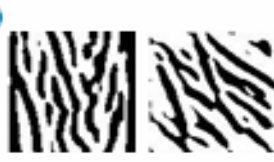

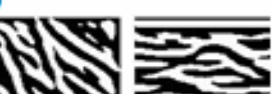

M

(g)

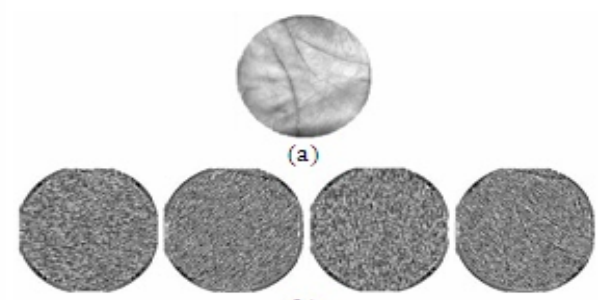

(b)

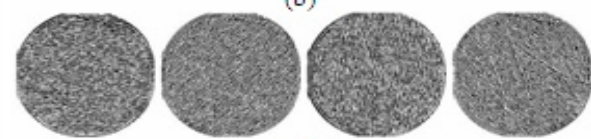

(c)

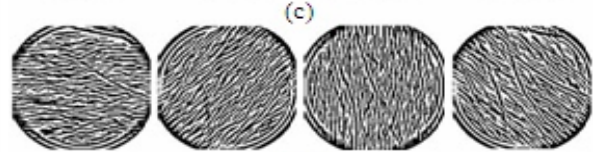

(d)

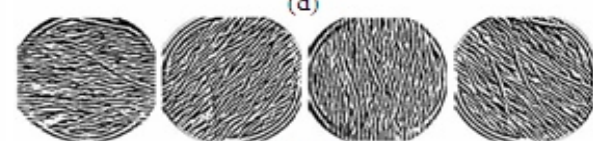

(e)
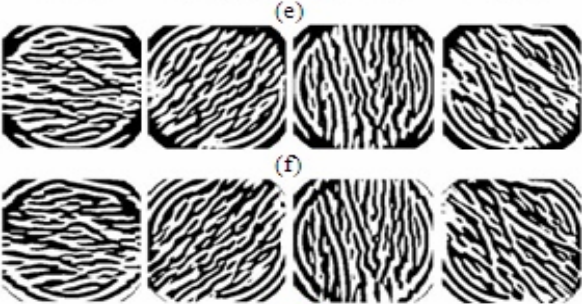

(g)

Fig. 5. Original image from DBI and DBII and their features generated by 12 filters listed in the Table1: (a)original image, (b), (d) and (f)real parts of features from Levels 1-3 filters, respectively, (c), (e) and (g)imaginary parts of features from Levels 1-3 filters, respectively

\subsubsection{Circular Gabor Filter}

Circular Gabor-based filter is a modified version of traditional Gabor filter. It is isotropic which means its characteristics are equal in every direction. It has been successfully used for invariant texture segmentation [15]. The circular Gabor filter is:

$$
c G(x, y, F, \sigma)=\frac{1}{2 \pi \sigma^{2}} \exp \left\{-\frac{\left(x^{2}+y^{2}\right)}{2 \sigma^{2}}\right\} \cdot \exp \left\{2 \pi i F\left(\sqrt{x^{2}+y^{2}}\right)\right\}
$$

where $\mathrm{F}$ is the central frequency of the circular Gabor filter. The frequency domain representation of the circular Gabor filter is given by

$$
F(u, v)=\frac{\sqrt{2 \pi}}{2} \alpha \exp \left(-\frac{\left(\sqrt{u^{2}+v^{2}}-F\right)^{2}}{2 \alpha^{2}}\right.
$$


where $\alpha=\frac{1}{2 \pi \sigma}$. The parameters are chosen from 3 sets of parameters listed in Table 2 . Palmprint presentation is exactly identical to section 2.2.1. Fig. 6 shows the features generated by the filter sets listed in Table 2 .

Table 2. The circular Gabor filter's parameters and their definitions

\begin{tabular}{lllll}
\hline Levels & No & Sizes & F & $\sigma$ \\
\hline 1 & 1 & $9 \times 9$ & 0.3666 & 1.4045 \\
2 & 5 & $17 \times 17$ & 0.1833 & 2.8090 \\
3 & 9 & $35 \times 35$ & 0.0916 & 5.6179 \\
\hline
\end{tabular}

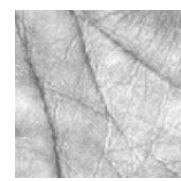

(a)

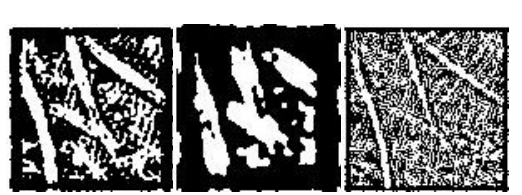

(b)

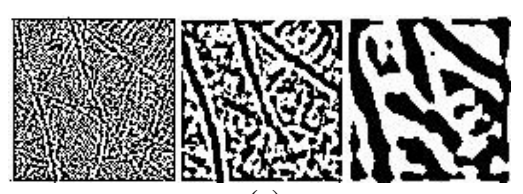

(c)

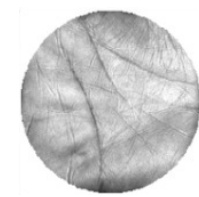

(a)

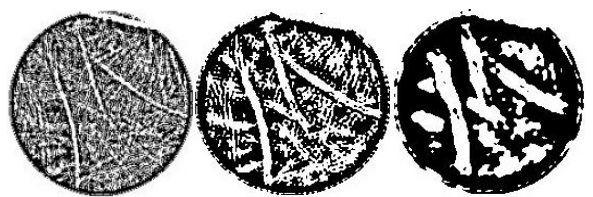

(b)

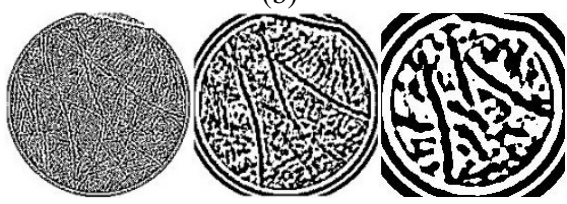

(c)

Fig. 6. Original image from DBI and DBII and their features generated by 3 filters listed in the Table2: (a) original image, (b) real parts of features from Levels 1-3 filters, (c), imaginary parts of from Levels 1-3 filters

\subsection{Feature Matching/Classification Procedures}

In order to describe clearly the matching process, each feature vector is considered as two 2-D feature matrices, real and imaginary. Palmprint matching is based on a normalized hamming distance. Let $\mathrm{P}$ and $\mathrm{Q}$ be two palmprint feature matrices. The normalized hamming distance can be defined as

$$
D_{0}=\frac{\sum_{i=1}^{n} \sum_{j=1}^{n}\left(P_{R}(i, j) \otimes Q_{R}(i, j)+P_{I}(i, j) \otimes Q_{I}(i, j)\right)}{2 N^{2}}
$$


where $P_{R}\left(Q_{R}\right)$ and $P_{I}\left(Q_{I}\right)$ are the real part and the imaginary part of $P(Q)$, respectively; the Boolean operator, " $\otimes$ ", is equal to zero if and only if the two bits, $P_{R(I)}(i, j)$ and $Q_{R(I)}(i, j)$ are equal and the size of the feature matrices is $\mathrm{N} \times \mathrm{N}$. It is noted that $D_{0}$ is between 1 and 0 . The hamming distance for perfect matching is zero. In order to provide translation invariance matching, Eq. (9) can be improved as

$$
D_{\min }=\min _{|s| S, \mid t<T} \frac{\sum_{i=\operatorname{maxx}(1+s)}^{\min N, N+s)} \sum_{j=\max (1+1)}^{\min N, N+t)}\left(P_{R}(i+s, j+t) \otimes Q_{R}(i, j)+P_{I}(i+s, j+t) \otimes Q_{I}(i, j)\right)}{2 H(s) H(t)}
$$

where $\mathrm{S}=2$ and $\mathrm{T}=2$ control the range of horizontal and vertical translation of a feature in the matching process, respectively and

$$
H(s)=\min (N, N+s)-\max (1+1+s)
$$

The hamming distance, $D_{\min }$ can support translation matching; nevertheless, because of unstable preprocessing, it is not a rotational invariant matching. Therefore, in enrollment mode, the coordinate system is rotated by a few degrees and then the subimages are extracted for feature extraction.

\section{Experimental Results and Analyses}

\subsection{Palmprint Database}

The proposed system has been tested on the PolyU-Palmprint-Database [16] that contains 7752 grayscale images corresponding to 386 different individuals. Around twenty samples from each individual were collected in two sessions, where around 10 samples were captured in both the first and second sessions, respectively. The size of all images is $384 \times 284$ with 75 dpi resolution. The square central parts of each image extracted with size $128 \times 128$ are named DBI and the circular central parts of each image extracted with average size of 100 pixels named DBII. Fig. 7 shows four typical images from this database.
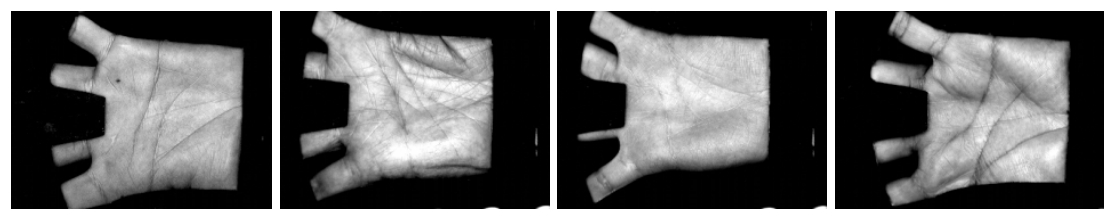

Fig. 7. Six typical images from the database

\subsection{Verification Test}

To obtain the verification accuracy of our palmprint system and better parameters for our system, 12 different sets of parameters listed in Table 1 and 3 different sets listed in Table 2 are used to test the method. Each of the images in DBI (DBII) is matched 
with all other palmprint images in the same database. A matching is counted as a correct matching if two palmprint images are collected from the same palm; otherwise it is an incorrect matching. The classification accuracy of the system based on Gabor features is presented in Tables 3 .

Table 3. Summary of accuracy rate for the different filters on two databases

\begin{tabular}{|c|c|c|c|c|c|c|c|c|c|}
\hline \multirow{3}{*}{\multicolumn{2}{|c|}{$\begin{array}{c}\text { Filter } \\
\text { No. }\end{array}$}} & \multicolumn{4}{|c|}{ DBI: Square sub-image } & \multicolumn{4}{|c|}{ DBII: Circle sub-image } \\
\hline & & \multicolumn{2}{|c|}{$\begin{array}{c}\text { Traditional Gabor } \\
\text { Filter }\end{array}$} & \multicolumn{2}{|c|}{$\begin{array}{l}\text { Circular Gabor } \\
\text { Filter }\end{array}$} & \multicolumn{2}{|c|}{$\begin{array}{c}\text { Traditional Gabor } \\
\text { Filter }\end{array}$} & \multicolumn{2}{|c|}{$\begin{array}{l}\text { Circular Gabor } \\
\text { Filter }\end{array}$} \\
\hline & & $\begin{array}{r}\text { FAR } \\
(\%)\end{array}$ & $\begin{array}{l}\text { FRR } \\
(\%)\end{array}$ & FAR $(\%)$ & FRR (\%) & $\begin{array}{c}\text { FAR } \\
(\%)\end{array}$ & $\begin{array}{l}\text { FRR } \\
(\%)\end{array}$ & FAR $(\%)$ & FRR $(\%)$ \\
\hline \multirow{4}{*}{ כ) } & 1 & 0 & 54.4 & \multirow{4}{*}{0} & \multirow{4}{*}{15.0} & 0 & 57.2 & \multirow{4}{*}{0} & \multirow{4}{*}{10.2} \\
\hline & 2 & 0 & 38.0 & & & 0 & 48.0 & & \\
\hline & 3 & 0 & 40.8 & & & 0 & 37.6 & & \\
\hline & 4 & 0 & 39.2 & & & 0 & 43.6 & & \\
\hline \multirow{4}{*}{ 常 } & 5 & 0 & 12.4 & \multirow{4}{*}{0} & \multirow{4}{*}{1.2} & 0 & 12.4 & \multirow{4}{*}{0} & \multirow{4}{*}{0.9} \\
\hline & 6 & 0 & 5 & & & 0 & 5.8 & & \\
\hline & 7 & 0 & 14.0 & & & 0 & 8.8 & & \\
\hline & 8 & 0 & 7.6 & & & 0 & 8.4 & & \\
\hline \multirow{4}{*}{$\frac{m}{d ! d}$} & 9 & 0 & 6.2 & \multirow{4}{*}{0} & \multirow{4}{*}{3.8} & 0 & 2.2 & \multirow{4}{*}{0} & \multirow{4}{*}{1.2} \\
\hline & 10 & 0 & 1.4 & & & 0 & 0.8 & & \\
\hline & 11 & 0 & 6.8 & & & 0 & 1.4 & & \\
\hline & 12 & 0 & 2 & & & 0 & 0.6 & & \\
\hline
\end{tabular}

\section{Conclusion}

Three key issues are involved in palmprint verification: preprocessing and segmentation, feature extraction and matching. The Gaussian filter was used for preprocessing. Whereas, noise reduction and the smoothing of palmprint images are done in preprocessing, palmprint segmentation involves correcting distortions and putting all palmprints under the same coordinate system so that the expected area of each palmprint (ROI) can be extracted for feature extraction and matching. Two kinds of palmprint segmentation approaches were presented. One was designed to extract $100 \times 100$ fixed size square area as the central part sub-image for the feature extraction, while the other one suggested using an inscribed circle (with the mean average radius of 100 pixel for database images).

Two different methods were employed to extract features from two sub-images of the central area of the palm. The first method used was the Gabor Filter feature extraction. Two kinds of Gabor Filter, traditional and circular, were used for this purpose, and two palmprint images are compared in terms of a criterion called hamming distance. The accuracy of $99.90 \%$ was obtained for identity verification through applying the circular Gabor filter on the square sub-images from the central area of the palm for the purpose of extracting feature. 


\section{References}

1. Kong, A., Zhang, D., Lu, G.: A Study of Identical Twins' Palmprints for Personal Authentication. In: Zhang, D., Jain, A.K. (eds.) ICB 2006. LNCS, vol. 3832, pp. 668-674. Springer, Heidelberg (2006)

2. Jain, A., Bolle, R., Pankanti, S.: Biometrics: Personal Identification in Networked Society. Kluwer Academic Publishers, Dordrecht (1999)

3. Miller, B.: Vital Signs of identity. IEEE Spectrum 2, 22-30 (1994)

4. Ross, A., Jain, A.K.: Multimodal Biometrics: An Overview. In: Proceedings of the 12th European Signal Processing Conference, pp. 1221-1224 (2004)

5. Lu, G., Zhang, D., Wang, K.: Palmprint Recognition using Eigenpalms Features. Pattern Recognition Letters 24, 1473-1477 (2003)

6. Li, W., Zhang, D., Xu, Z.: Palmprint Identification by Fourier Transform. International Journal of Pattern Recognition and Artificial Intelligence 16, 417-432 (2003)

7. Funada, J., Ohta, N., Mizoguchi, M., Temma, T., Nakanishi, K., Murai, A., Sugiuchi, T., Wakabayashi, T., Yamada, Y.: Feature Extraction Method for Palmprint Considering Elimination of Creases. In: Proceedings of the 14th International Conference on Pattern Recognition, vol. 2, pp. 1849-1854 (1998)

8. Zhang, D., Shu, W.: Two Novel Characteristics in Palmprint Verification: Datum Point Invariance and Line Feature Matching. Pattern Recognition 32, 691-702 (1999)

9. Wu, X., Wang, K., Zhang, D.: Fuzzy Directional Element Energy Feature (FDEEF) based Palmprint Identification. In: Proceedings of the 16th International Conference on Pattern Recognition, vol. 1, pp. 95-98 (2002)

10. Han, C.C., Cheng, H.L., Lin, C.L., Fan, K.C.: Personal Authentication using Palmprint Features. Pattern Recognition 2, 281-371 (2003)

11. Wu, X., Zhang, D., Wang, K.: Fisherpalms based Palmprint Recognition. Pattern Recognition Letters 24, 2829-2838 (2003)

12. Li, W., You, J., Zhang, D.: Texture-based Palmprint Retrieval using a Layered Search Scheme for Personal Identification. IEEE Transactions on Multimedia 7, 891-898 (2005)

13. Kong, W.K., Zhang, D., Li, W.: Palmprint Feature Extraction using 2-D Gabor Filters. Pattern Recognition 10, 2339-2347 (2003)

14. You, J., Li, W., Zhang, D.: Hierarchical Palmprint Identification via Multiple Feature Extraction. Pattern Recognition 35, 847-859 (2002)

15. Zhang, J., Tan, T.: Rotation Invariant Texture Segmentation via Circular Gabor Filters. In: IEEE International Conference on Pattern Recognition (2002)

16. Biometrics Research Centre (BRC), http: / /www4 . comp.polyu. edu.hk/ biometrics /

17. Wu, P.S., Li, M.: Pyramid Edge Detection based on Stack Filter. Pattern Recognition Letters 18, 329-348 (1997) 